Monatsschrift f. Geburtshülfe u. Gynäkologie 1918;47:I-V

\title{
Contents, Vol. 47, 1918
}

\section{Inhalts - Verzeichnis.}

Orîginal-Arbeiten. Seit $\beta$

Abel, G., Ein Fall von Hydrocele feminina 366

A h 1 fe 1 d, E., Wrisberg, Mayor, de Kergeradec, Zur Ge-

schichte und Entwicklung der geburtshülf lichen Aus-

kultation

187

B o $1 \mathrm{~d} \mathrm{t}$, H. J., Chlorzink bei uterinen Blutungen, besonders

wenn dieselben durch kleine interstitielle Myome, Metro-

endometritis oder Fibrosis uteri entstanden sind . . . 522 B u s e h , II., Zur Indikationsstellung

des künstlichen Aborts 93 Franz, Tb., Zur Physiologie und Leitung der III. Ge-

burtsperiode 217

F r ü h o 1 z, B., Die Behandlung der Gesichtslage mit nach

hinten gerichtetem Kinn 431

Fürbringer, Zur Erage der Sexualperiodizität beim

weiblichen Geschlecht

Heynemann, TL, Zum Wesen und zur Behandlung der

Eklampsie $\quad 425$

Jolly, R., Über Zufälle bei Laparotomien 525

K e i 11 e r, H., Über Doppelkarzinome des Uterus (Hierzu

Taf. Ill-IX) 285

K ü s t e r , H., Über Mesenterialzysten 145

Über Gallertbauch (das sog. Pseudomyxoma psritonei) 477

Lindig, $\mathrm{P} a \backslash \backslash 1$, Die Brustdrüsensekretion beim $\mathrm{Neu}^{\wedge}$

geborenen 534

Martin, A., Der sogenannte Wigand- A. Martin-v. Winckel-

sche Handgriff

21

Moraller,F., Über blutende Mamma mit besonderer Be-

rücksichtigung des Cystadenoma mammae. (Hierzu

Tafel I-II) ,...." 107

Mönch, G., siehe Schmidt, E. und G. Mönch. Ramsauer, L., Ein Beitrag zur Frage der Radiumund

Mesothoriumtherapie bei Karzinomen des weiblichen

Genitale $\quad 153,253$

- IV -

Seite

Eöhmann, F., Über den Einfluß der Ernährung auf die

Sekretion der Milchdrüse 455

Rübsamen,W., Nierenbeckenplastik nach Fenger bei ge-

platzter Hydronephrose $\quad 505$ 
Ruge, Georg, Haarrichtungslinien im Bereiche des

Mammarapparates bei mensch.lich.en Embryonen ... 2 Ruge I, Carl, Fehldiagnosen und zufällige Befunde . . 27

Ruge II, Carl, Über Lebereklampsie 37

Rukop, E., Verfahren zur Einschränkung der manuellen

Placentalösung 103

Schmidt,E.u. Möncb , G., Zur Ätiologie der angebore-

nen allgemeinen Wassersucht 368

Schmidt, Phil ipp, Über den Einfluß der Kriegsernäh-

rung auf das Körpergewicht der Neugeborenen .... 390 Schroder, Rob., Die

Echinokokkenerkrankung in der

Gynäkologie und Geburtshülfe : . . 509-

W e i 11 , G., Ergotin als Herzmittel und Diureticum . . 250 Winter, G., Sollen wir

Bevölkerungspolitik treiben? . .351 Wulff, Hans, Ein Fall von Verblutungstod am siebenten

Wochenbetttage durch Ruptura uteri incompleta . . .462

Über Beinhalter bei operativen Entbindungen in der

Praxis 531

Beriehte.

Der Operateur und sein Recht im neuen Strafgesetzbuch. Von

Dr. Hans Lieske in München-Ganting 61

Die Entwicklung der Strahlentherapie im Jahre 1917.

Sammelbericht von A. Martin in Berlin 544

Die deutschen Frauenkliniken im siebenten Kriegssemester 76, 280

Bericht über die französische geburtshülflich-gynäkologische

Literatur der Jahre 1914, 1915, 1916. Von Dr. F.

Mandach in Zurich $\quad 49,270$

Übersicht über die Holländische Literatur (1915). Von Th. H. vandeVelde und J. H. Pad t berg in Haarlem . . 406

Paul Ruge zum 70. Geburtstage 1

W. A. Freund $\uparrow$. Von R. Mü Her h eim in Berlin .... 171

Bibliographle von W. A. Freund. Von Dr. v. d. V e $1 \mathrm{~d}$ e in

Düsseldorf 177

$-\mathrm{V}$ -

Seite

Vereins- und Literaturbeilage.

Gesellschaft für Geburtshülfe und Gynäkologie in Berlin.

Sitzung vom 14. XII. 1917, 11. I. 1918, 22. II. 1918,

15. III. 1918, 82, 281, 494, 573 Gynäkolog $1 / 8$ che Gesellschaft in Breslau. Sitzung vom 6. XI.

1917 und 5. II. 1918, Nachtrag 180, 487, 558

Münchener gynäkologische Gesellschaft. Sitzung vom 24. V.

1917, b. VII. $1917 \quad 559$

Geburtslmlflich-gynäkologische Gesellschaft in Wien. Sitzung

vom 13. XI. 1917, 18. I. 1918, 12. II. und 26. II. 1918

12. HI. 1918, 83, 419, 495, 569 Geburtshülflich-gynäkologische Mitteilungen aus Medizinischen Gesellschaften und Vereinen 86,183, 283, 422, 503, 574

Buchanzeigen 87, 183 
Literaturverzeichnis $\quad 89,184,284,422,504,574$

Personalien .81, 179, 280, 418, 486, 571, 558

Sachregister 575

Namen register _. .579 